\title{
Dewetting of Polymer Blends and Formation of Phase-Separated Polymer Domes
}

\author{
Olaf Karthaus* and Yuji Kiyono \\ Chitose Institute of Science and Technology, Bibi 65-758, Chitose 066-8655, Japan
}

(Received 9 March 2006; Accepted 14 March 2006; Published 17 March 2006)

\begin{abstract}
Phase-separated polymer droplets with micron-size diameter and nanometer-size height were prepared by casting a dilute solution of two polymers, polystyrene and poly(methylmethacrylate). Staining with an electron acceptor allowed us to distinguish between the two polymers by fluorescence microscopy. [DOI: 10.1380/ejssnt.2006.270]
\end{abstract}

Keywords: Adhesion; Photoluminescence; Surface segregation; Surface structure, morphology, roughness, and topography; Wetting; Polymers

\section{INTRODUCTION}

Preparation of heterogeneous nano- and microstructures by self-assembly and self-organization is one of the research interests in surface science with a large potential. Soft matter, like polymers are well suited to modify and functionalize surface properties over a wide range. Applications range from control of cell adhesion to the preparation of lithographic masks for semiconductor electronics.

Spin-coating of block-copolymers or polymer blends has been used to prepare periodic structures on patterned [1, $2]$, and non-patterned substrates [3]. On the other hand, dewetting has been used to form periodic structures of polymer microdomes on substrates [4].

Here we study a polymer blend to fabricate a new morphology of phase-separated structures on substrates: isolated polymer microdomes in which the upper parts consist of polystyrene and the lower part of poly(methylmethacrylate).

\section{EXPERIMENTAL}

Polystyrene (PS; $\left.M_{w}=230,000 \mathrm{~g} / \mathrm{mol}\right)$ and poly(methylmethacrylate) (PMMA; $M_{w}=380,000$ $\mathrm{g} / \mathrm{mol}$ ) were purchased from Aldrich. Tetracyanoquinodimethane (TCNQ) was purchased from TCI and used without further purification. Glass substrates (Matsunami Neo) with a thickness of $0.15 \mathrm{~mm}$ were cleaned in an ozone cleaner. Fluorescence images were taken with a fluorescence microscope (Olympus BX-51), equipped with a color CCD camera (SPOT RT-CL, Diagnostics Inc.). $10 \mathrm{mg}$ of each polymer were dissolved in $10 \mathrm{ml}$ of ethylacetate and $1 \mathrm{~mol} \%$ (in respect to the repeat unit of the polymers) of TCNQ was added. The solution was placed in the $0.5 \mathrm{~mm}$ air gap between the substrate and a glass-roller apparatus. The design of the apparatus is described in detail elsewhere [5]. Fluorescence spectra of polymer films were recorded by a home-built fluorescence microspectrometer. A He-Cd laser (excitation $442 \mathrm{~nm}$ ) was used and the fluorescence was analyzed by a cooled CCD camera attached to a polychromator equipped with

\footnotetext{
*Corresponding author: karthaus@photon.chitose.ac.jp
}

a grating of 150 lines/cm.

\section{RESULTS}

The glass substrate became slightly turbid after dewetting, indicating the presence of surface inhomogeneities. Scanning electron microscopy (SEM) showed the presence of polymer domes with a diameter of 1-20 $\mu \mathrm{m}$. The mechanism of dome formation from dilute solution in a volatile solvent has been described in detail in a previous publication and involves a fingering instability at the edge of the evaporating solution [4]. The contact angle of the resulting polymer droplets with the glass substrate is around 5 degrees which is reasonable for dewetted polymers [4], as can be seen in the SEM picture in Fig. 1. In the case of amorphous polymers and dendrimers $[6]$ the dome surface is featureless and spherical. In the present case of a polymer blend, step at around half the dome height can be seen. The step is surrounded by a flat area, the step itself has a contact angle of about 8 degrees, which is again a reasonable value for a dewetted polymer. Since PS and PMMA are not miscible, this step is most likely due to the phase boundary between a PS-rich and PMMA-rich phase. It is difficult to calculate the exact volume ratios from the SEM picture alone, especially because the in-

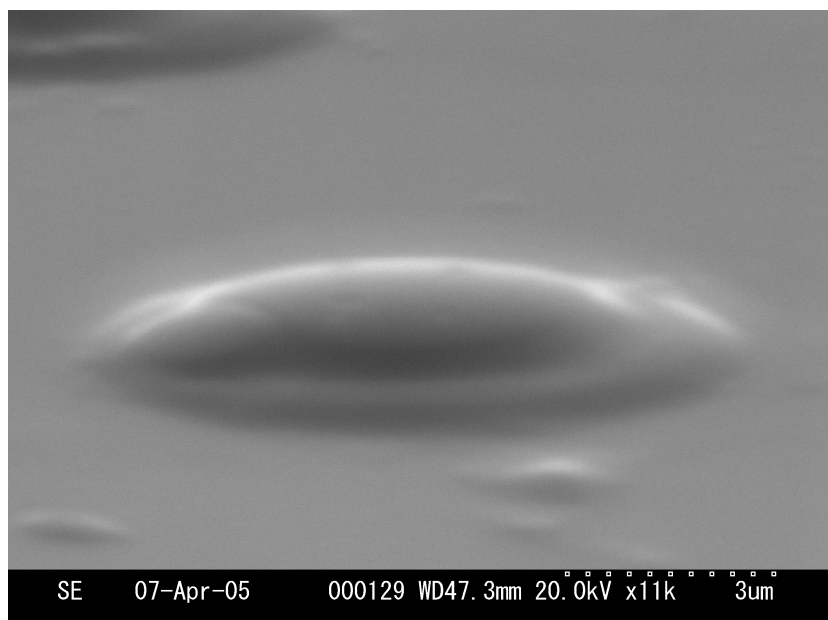

FIG. 1: Scanning electron micrograph of a phase separated microdome of polystyrene and poly(methylmethacrylate). 


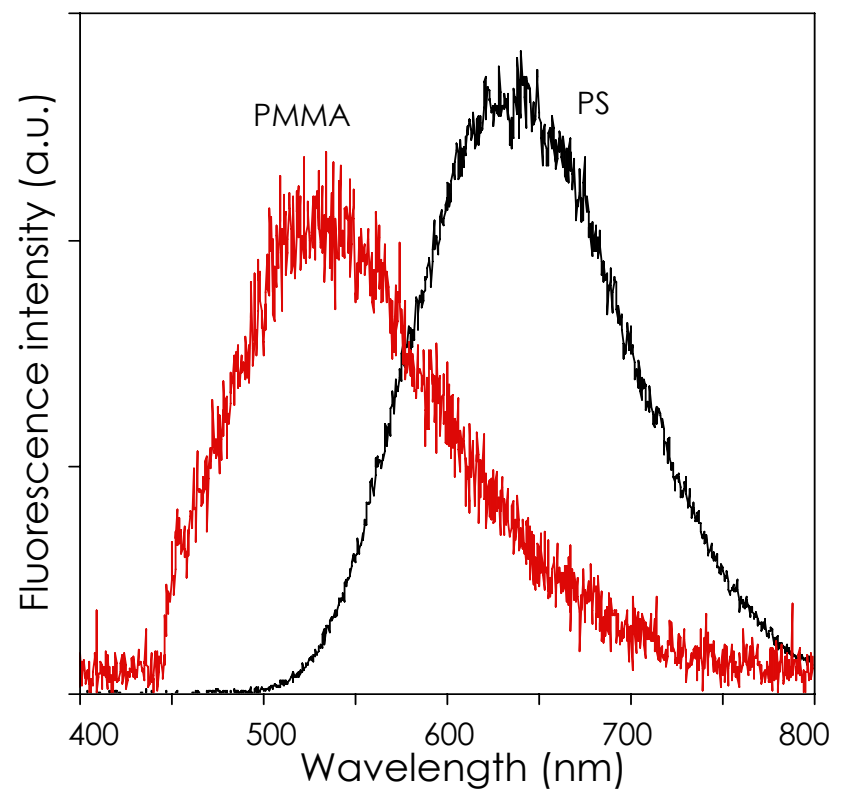

FIG. 2: Fluorescence spectra of spin coated films of poly(methylmethacrylate) (PMMA) doped with 1 mol\% TCNQ (red curve), and polystyrene doped with 1 mol\% TCNQ (black curve).

terface between a PMMA film and dewetted polystyrene droplets is most likely not horizontal. It was already reported that dewetted PS droplets extend into a PMMA base film $[7,8]$. Nevertheless, the volumes of the polymer dome above the step and below seem to be similar. This is another evidence for the phase separation and the formation of a straight interface between the two immiscible polymers.

More evidence for a phase separation comes from the fluorescence image of the domes.

In order to determine which of the two phases is polystyrene, and which is PMMA, both polymers were doped with TCNQ, a strong electron acceptor. It is known that TCNQ forms a charge-transfer complex with polystyrene, because of the electron-rich benzene ring in PS and the possibility to form a sandwich complex of one TCNQ intercalated between two PS benzene rings. The electronic interaction between electron donor and acceptor leads to a shift in the HOMO and LUMO energy levels and red fluorescence spectrum is observed in PS films doped with TCNQ, as can be seen in Fig. 2. The fluorescence maximum is at $638 \mathrm{~nm}$. This CT interaction is not possible in TCNQ-doped PMMA, which shows a green fluorescence with a fluorescence maximum at 530 $\mathrm{nm}$. Fluorescence microscopy of the dewetted dome structures (Fig. 3) show that all the domes have a central red fluorescence that is surrounded by a ring of green fluorescence. The location of the edge of the red fluorescent spot in comparison with width of the green fluorescent ring indicates that the red spot coincides with the upper polymer layer in Fig. 1. The green ring corresponds to the image of the lower polymer phase when viewed vertically.

The fact that PS forms a cap on a base layer of PMMA can be reasonably explained by the fact that the polar ester groups of PMMA interact more with the polar Si$\mathrm{OH}$ or Si-O-Si groups of the glass surface that the apolar polystyrene. Furthermore, the surface tension of PS is around $43.5 \mathrm{mN} / \mathrm{m}$, as compared to $49 \mathrm{mN} / \mathrm{m}$ for PMMA [9], thus the lower surface tension PS tends to accumulate at the polymer-air interface [7, 8].

Even though the polymer domes formed on the glass surface by dewetting are not homogeneously spaced and have a wide size distribution, all show the phase-separated structure with a PS cap on a PMMA base layer. Domes as small as $1 \mu \mathrm{m}$ and as large as $10 \mu \mathrm{m}$ all show the same morphology. Larger domes, as the ones partially seen at the right edge of the fluorescence micrograph, may show deviation from the spherical shape and the PS cap may be split into several islands [10].

The ease of the sample preparation is intriguing. The applicability to other polymer combinations is interesting for the preparation of functional mesoscopically ordered self-assembled polymer structures.

\section{Acknowledgments}

The present work is supported by the Grant-in-Aid for Scientific Research (KAKENHI) in Priority Area Molecular Nano Dynamics from Ministry of Education, Culture, Sports, Science and Technology, Japan.

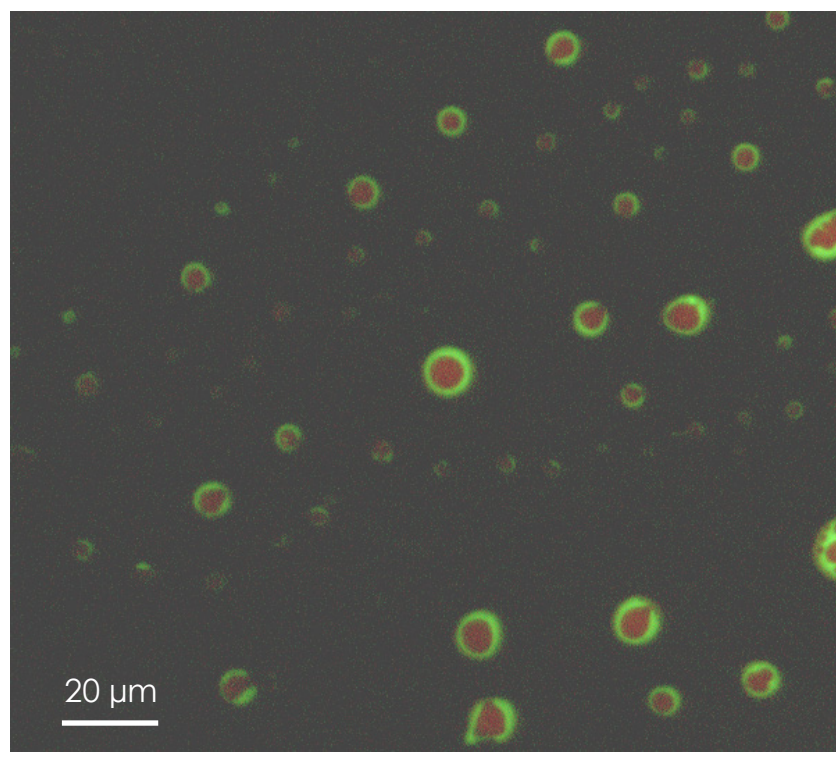

FIG. 3: Fluorescence micrograph of a dewetted sample of PS and PMMA doped with TCNQ. The excitation wavelength was 440-480 nm.
[1] K. Fukunaga, H. Elbs, G. Krausch, Langmuir 16, 3474 (2000).
[2] K. C. Daoulas, M. Muller, M. P. Stoykovic, S.-M. Park, Y. J. Papakonstantopoulos, J. J. de Pablo, P. F. Nealey, 
H. H. Solak, Phys Rev. Lett. 96, 036104 (2006).

[3] S. Walheim, E. Schaffer, J. Mlynek, U. Steiner, Science 283, 520 (1999).

[4] O. Karthaus, L. Grasjo, N. Maruyama, and M. Shimomura, Chaos 9, 308 (1999).

[5] K. Kaga, K. Okamoto, T. Echizen, O. Karthaus, K. Nakajima, Kobunshi Ronbunshu 60, 752 (2003).

[6] J. Hellmann, M. Hamano, O. Karthaus, K. Ijiro, M. Shimomura, M. Irie, Jpn. J. Appl. Phys. 37, L816 (1998).

[7] X. Rui, Z. Song, S. Jing, D. Tian, Polym. J. 37, 560
(2005).

[8] C. Yuan, M. Ouyang, J. T. Koberstein, Macromolecules 32, 2329 (1999).

[9] www.igb.fraunhofer.de/www/GW/GrenzflMem/ Grenzflaechen/gf-physik/en/GFphysPolymOberfl.es.html as of March 9, 2006.

[10] Y. Li, Y. Yang, F. Yu, L. Dong, J. Polym. Sci. B 44, 9 (2006). 